\title{
Two Streams of Attention-Dependent $\beta$ Activity in the Striate Recipient Zone of Cat's Lateral Posterior-Pulvinar Complex
}

\author{
Andrzej Wróbel, ${ }^{1,2}$ Anaida Ghazaryan, ${ }^{1}$ Marek Bekisz, ${ }^{1}$ Wojciech Bogdan, ${ }^{1}$ and Jan Kamiński ${ }^{1,2}$ \\ ${ }^{1}$ Department of Neurophysiology, Nencki Institute of Experimental Biology, 02-093 Warsaw, Poland, and 2Department of Psychology, Warsaw School of \\ Social Psychology, 03-815 Warsaw, Poland
}

Local field potentials from different visual cortical areas and subdivisions of the cat's lateral posterior-pulvinar complex of the thalamus (LP-P) were recorded during a behavioral task based on delayed spatial discrimination of visual or auditory stimuli. During visual but not auditory attentive tasks, we observed an increase of $\beta$ activity $(12-25 \mathrm{~Hz})$ as calculated from signals recorded from the caudal part of the lateral zone of the LP-P (LPl-c) as well as from cortical areas 17 and 18 and the complex located at the middle suprasylvian sulcus (MSS). This $\beta$ activity appeared only in the trials that ended with a successful response, proving its relationship to the mechanism of visual attention. In contrast, no enhanced $\beta$ activity was observed in the rostral part of the lateral zone of the LP-P and in the pulvinar proper. Two subregions of LPl-c (ventromedial and dorsolateral) were distinguished by visually related, attentional $\beta$ activity of low (12-18 Hz) and high $(18-25 \mathrm{~Hz})$ frequencies, respectively. At the same time, area 17 exhibited attentional activation in the whole $\beta$ range, and an increase of power in low-frequency $\beta$ was observed in the medial bank of MSS, whereas cortical area 18 and the lateral bank of the MSS were activated in the high $\beta$ range. Phase-correlation analysis revealed that two distinct corticothalamic systems were synchronized by the $\beta$ activity of different frequencies. One comprised of cortical area 17, ventromedial region of LPl-c, and medial MSS, the second involved area 18 and the dorsolateral LPl-c. Our observations suggest that LPl-c belongs to the wide corticothalamic attentional system, which is functionally segregated by distinct streams of $\beta$ activity.

Key words: visual attention; corticothalamic system; $\beta$ oscillations; phase synchronization; cat

\section{Introduction}

It is widely agreed that the descending projection from layer 6 of the visual cortex $(\mathrm{VCx})$ plays a modulatory role in the transmission of retinal information through the dorsal lateral geniculate nucleus, a "first-order relay" (Sherman and Guillery, 2002). One putative function of this feedback loop might be frequency dependent, variable gain regulation (Lindström and Wróbel, 1990; Waleszczyk et al., 2005) used during attentive visual processing in the $\beta$ range (Bekisz and Wróbel, 1993; Wróbel, 2000). The other, less well recognized corticothalamic visual circuits involve layer 5 pyramidal cells of the cortex and the extrageniculate thalamus [in the cat: lateral posterior-pulvinar (LP-P) nuclear group]. Because the cortical input seems to be a crucial driver for cells in the extrageniculate visual thalamus (Chalupa, 1991; Casanova et al., 1997), this nuclear group is often referred to as a "higher-order thalamic relay” (Feig and Harting, 1998; Grieve et al., 2000; Sherman and Guillery, 2002). Specifically, striate cortex innervates the caudal part of the lateral zone of the cat LP-P (Guillery and

Received Sept. 14, 2006; revised Jan. 22, 2007; accepted Jan. 22, 2007.

This work was supported by State Committee for Scientific Research Grants 6 P05A 09020 and N401 14631/3239. We thank Joanna Smyda for excellent help in animal training and Wojciech Borkowski for securing the technical part of experiments.

Correspondence should be addressed to Andrzej Wróbel, Nencki Institute of Experimental Biology, 3 Pasteur Street, 02-093 Warsaw, Poland. E-mail: wrobel@nencki.gov.pl.

D0I:10.1523/JNEUROSCI.4004-06.2007

Copyright $\odot 2007$ Society for Neuroscience $\quad$ 0270-6474/07/272230-11\$15.00/0
Sherman, 2002) and in turn receives reciprocal input back from this region (Abramson and Chalupa, 1985; Miceli et al., 1991). The question arises whether the recurrent circuitry of higherorder visual processing might use activation in the $\beta$-frequency range for attention-related modulation as observed previously in the "first-order" corticogeniculate loop (Wróbel, 2000).

All previous behavioral investigations undertaken to establish the functional role of the extrageniculate visual thalamus were performed on monkeys. First observations suggested that impairments in the acquisition of visual pattern discrimination tasks observed after inferior pulvinar lesions may involve attentional deficits (Gould et al., 1974; Chalupa et al., 1976). Subsequent studies revealed that pulvinar complex is involved in spatial attention tasks (Petersen et al., 1987) and may suppress inputs related to irrelevant events (Desimone et al., 1990; Robinson and Petersen, 1992) and proposed its major role in attentive behavior and visual salience (Chalupa, 1991; Shipp, 2004).

Both in the cat and monkey, it has been proposed that the extrageniculate visual thalamus provides a link between primary and higher-order visual areas by means of "re-entrant" circuitry (Olshausen et al., 1993; Guillery, 1995; Jones, 2001; Sherman and Guillery, 2002; Shipp, 2004), which may subsequently spread attended visual information within the network (Chalupa et al., 1976; Petersen et al., 1987; Bender and Youakim, 2001; Casanova et al., 2001).

To further define the functional role of the lateral posterior- 

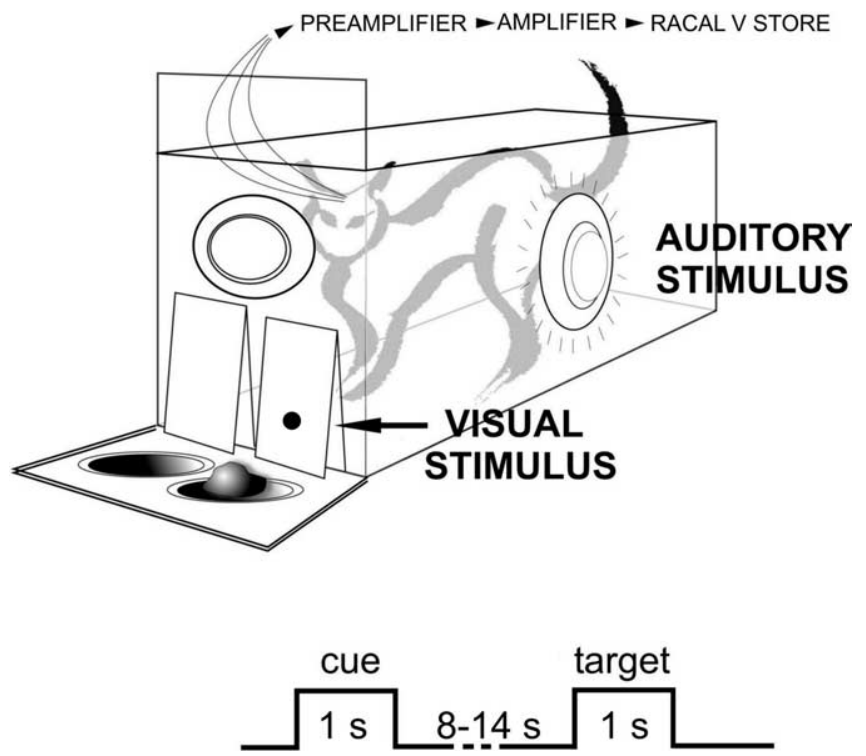

Figure 1. The attention paradigm used in this study. The visual and auditory trials started with cue stimuli of a given modality: a short diffuse flash behind the frontal wall or a white noise delivered from the frontal loudspeaker. In a given modality trial, the animal anticipated the target stimulus for 8-14s, with no stimulation present until a small spot of light or white noise from the side loudspeaker was delivered. Two seconds later, the transparent screen was removed, and the cat was allowed to open the indicated door for a food reward. Visual trials $(16-20)$ were randomly intermingled with auditory (16-20) trials. During the experiment, the cage was covered by a removable ceiling. See Materials and Methods for details.

pulvinar complex of the cat in visual processing, we analyzed attention-related changes of local field potentials recorded from this nuclear group and from interconnected primary and higherorder cortical visual areas. Among the latter, the middle suprasylvian region was chosen because of its strong interconnections with the LP-P (Garey et al., 1991; Payne and Lomber, 2003; Huppe-Gourgues et al., 2006) and its involvement in visually guided behavior (Kiefer et al., 1989; Shupert et al., 1993; Rudolph and Pasternak, 1996; Ouellette et al., 2004). The phasecorrelation analysis between the recorded signals was used to determine the dynamics of functional connections within the network during attentive behavior.

Preliminary results have been published previously in abstract form (Wróbel et al., 2005).

\section{Materials and Methods}

All experimental procedures were approved by the Ethical Commission at the Nencki Institute.

Behavioral paradigm. Ten adult, castrated male cats weighing 3-3.5 kg were used. Each cat was trained to discriminate the spatial position of a reward based on visual or auditory cues in intermingled trials. Training was performed in a wooden box $(20 \times 45 \times 45 \mathrm{~cm})$ with right and left semitranslucent doors on the front wall and a transparent screen preventing the animal's access to feeders placed behind the doors (Fig. 1). Visual stimuli were presented through the frontal doors and auditory ones through loudspeakers mounted behind the frontal, left, and right walls. Additionally, data from four cats trained with a similar experimental paradigm in our previous experiment (Bekisz and Wróbel, 2003) were added to increase the cortical sample.

The animals were trained in an "anticipatory attention paradigm" within the cage dimly lit at low mesopic range $\left(0.05 \mathrm{~cd} / \mathrm{m}^{2}\right)$. The visual and auditory trials started with cue stimuli of a given modality: a short diffuse flash of light back-projected on the doors $\left(0.6 \mathrm{~cd} / \mathrm{m}^{2}\right.$ and $1 \mathrm{~s}$ duration) or a white noise delivered from the frontal loudspeaker (60 dB and $1 \mathrm{~s}$ duration). During the trials, the animal anticipated the target stimulus for $8-14 \mathrm{~s}$ (the duration of this period changed randomly from trial to trial) with no conditional stimuli present. The visual target stimulus was a small spot of light $\left(0.5 \times 1^{\circ}\right.$ of $\sim 1.6 \mathrm{~cd} / \mathrm{m}^{2}$ intensity and $1 \mathrm{~s}$ duration) that appeared afterward at one of the doors. Two seconds later, the transparent screen was removed and the cat was allowed to open the indicated door for a food reward. Visual trials were randomly intermingled with auditory trials that ended with a target (white noise of $\sim 50 \mathrm{~dB}$ for $1 \mathrm{~s}$ ) delivered from a loudspeaker mounted behind the left or right wall, indicating the door that should be opened for a reward. In each experimental session, the visual and auditory trials were repeated 16 or 20 times each, in random order. The learning procedure started with the visual task. The auditory trials were introduced after the animal reached $90 \%$ performance level in the visual task. Intensity of visual and auditory target stimuli was adjusted to attain an error rate of $\sim 10 \%$. The training procedure ended when animals differentiated visual and auditory tasks with $90 \%$ accuracy during three successive experimental days.

Animal preparation. After the training procedure, electrode implantation was performed under pentobarbital sodium anesthesia $(30 \mathrm{mg} / \mathrm{kg}$; Nembutal sodium solution; Abbot Laboratories, Abbott Park, IL) with subsequent supplementary doses ( $3 \mathrm{mg} / \mathrm{kg}$ when required) using premedication with $0.2 \mathrm{mg} / \mathrm{kg}$ combelen and $0.3 \mathrm{ml}$ of atropine sulfate $(0.05 \%$; Warszawskie Zaklady Farmaceutyczne Polfa, Warsaw, Poland). One to three recording electrodes $(80-130 \mu \mathrm{m}$ exposed tip; tungsten wire coated in lacquer; $50-100 \mathrm{k} \Omega$ impedance at $1 \mathrm{kHz}$ ) were inserted under electrophysiological control (recording the swish to hand-held visual stimuli) into three divisions of lateral posterior-pulvinar complex (caudal and rostral parts of the lateral zone and the pulvinar proper). The remaining electrodes were implanted $\sim 1 \mathrm{~mm}$ apart from each other into visual cortical areas 17 and 18, where the more posterior locations were chosen to be close to the superficially available representation of area centralis. Electrodes were also placed in one of the so-called "lateral suprasylvian visual cortex" areas, which are predominantly located within middle suprasylvian sulcus (MSS) at its medial [posteromedial and anteromedial lateral suprasylvian visual areas (PMLS and AMLS)] and lateral [posterolateral and anterolateral lateral suprasylvian visual areas (PLLS and ALLS)] banks (Tusa et al., 1981; Grant and Hilgetag, 2005). All recording sites in each cat are listed in Tables 1 and 2. Two recording electrodes were also inserted in the left lateral geniculate nucleus (LGN) and/or perigeniculate nucleus for purposes of another project. All electrodes were placed in the left hemisphere, and the cortical electrodes were positioned at a depth of about layer 4 . All electrodes and a connecting plug were fixed on the skull using dental cement, and recordings started 1-2 weeks after surgery.

After completion of all recording sessions, cats were anesthetized with Nembutal, and small electrolytic lesions ( $5 \mu \mathrm{A}$ for $15 \mathrm{~s}$ positive $\mathrm{DC}$ ) were made to mark electrode tip positions. The animals were then killed with a lethal dose of pentobarbital and perfused with $4 \%$ paraformaldehyde in $0.1 \%$ phosphate buffer for subsequent histological verification. In two animals, every third section was stained to reveal acetylcholinesterase activity to better distinguish the three major zones in LP-P (Berson and Graybiel, 1983). The physiologically estimated retinotopic positions corresponding to recording sites were finally verified from postmortem anatomical analysis with reference to the maps provided by Hutchins and Updyke (1989) for LP-P and Tusa et al. (1981) for visual cortical areas. The retinotopy of the MSS recording sites was not confirmed physiologically, and we therefore relied only on the histology matched to the maps defined by others (Tusa et al., 1981; Grant and Shipp, 1991). Taking into account the large distance from which local field potential (LFP) sources can be recorded $(0.4 \mathrm{~mm})$, the possible error of such evaluation was within the limits of our recording method.

Recording and data analysis. Monopolar LFPs amplified 1000 times (with bandwidth of $1 \mathrm{~Hz}$ to $5 \mathrm{kHz}$ ) were recorded throughout the whole experimental session on an FM magnetic tape recorder (Racal V-Store; Racal Recorders, Southampton, UK). Low-pass-filtered data (3 dB amplitude attenuation at $100 \mathrm{~Hz} ; 24 \mathrm{~dB} /$ octave; Bessel analog filter) were digitized with a $400 \mathrm{~Hz}$ sampling rate. The signal analysis was limited to LFPs recorded between the cue and the target stimuli (i.e., 8-14 s). Fast Fourier Transform (FFT) amplitude spectra were calculated for each trial for a number of consecutive time epochs. Each epoch was 512 samples (1.28 s) long and shifted by 240 samples ( $~ 50 \%$ overlap) from the pre- 
Table 1. List of experimental animals with location of electrodes, their corresponding retinotopic positions, and frequency ranges of significant increases of amplitude spectra in visually attentive trials.

\begin{tabular}{|c|c|c|c|c|c|c|c|c|c|c|c|}
\hline & & & $\mathrm{VCx}$ area 1 & & & & VCx area 1 & & & Middle & asylvian banks \\
\hline & LPI-C & & Posterior & 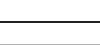 & $\longrightarrow$ & anterior & Posterior & 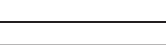 & $\longrightarrow$ anterior & Medial & Lateral \\
\hline $\begin{array}{l}\text { Cat } 7 \\
\text { (vm LP1-c) }\end{array}$ & $\begin{array}{l}5 /+5 \\
12-19 \\
36-37\end{array}$ & $42-43$ & $\begin{array}{l}0 /+5 \\
12-17 \\
37-43\end{array}$ & & & $\begin{array}{l}0 /-5 \\
12-19 \\
31-33\end{array}$ & & & & & \\
\hline $\begin{array}{l}\text { Cat } 8 \\
\text { (dl LP1-c) }\end{array}$ & $\begin{array}{l}0 /+10 \\
20-25\end{array}$ & & $\begin{array}{l}0 /+2.5 \\
15-20\end{array}$ & & & $\begin{array}{l}0 /-2.5 \\
15-18\end{array}$ & & & & & \\
\hline $\begin{array}{l}\text { Cat } 9 \\
\text { (vm LP1-c) }\end{array}$ & $\begin{array}{l}5 /+5 \\
12-16\end{array}$ & $\begin{array}{l}2 / 1 \\
12-15\end{array}$ & & & $\begin{array}{l}0 /-2.5 \\
12-16\end{array}$ & $\begin{array}{l}0 /-5 \\
12-17\end{array}$ & & $\begin{array}{l}5 /-5 \\
18-23 \\
38-42 ; 44-48\end{array}$ & $\begin{array}{l}5 /-10 \\
19-28 \\
30-32\end{array}$ & $12-15$ & \\
\hline $\begin{array}{l}\text { Cat } 10 \\
\text { (dl LP1-c) }\end{array}$ & $\begin{array}{l}10 /+5 \\
16-21\end{array}$ & & & & $\begin{array}{l}0 /-5 \\
13-18 \\
36-37\end{array}$ & $\begin{array}{l}5 /-10 \\
13-22\end{array}$ & & $\begin{array}{l}5 /-15 \\
18-23 \\
40-41\end{array}$ & $\begin{array}{l}20 /-15 \\
17-23 \\
37-38 ; 40-42\end{array}$ & $14-18$ & \\
\hline $\begin{array}{l}\text { Cat } 12 \\
\text { (dl LP1-c) }\end{array}$ & $\begin{array}{l}20 /-5 \\
18-22\end{array}$ & & & & $\begin{array}{l}0 /+2.5 \\
12-24\end{array}$ & $\begin{array}{l}0 /-4 \\
19-24 \mathrm{~Hz}\end{array}$ & $\begin{array}{l}0 /-2.5 \\
19-21 \\
37-42\end{array}$ & & $\begin{array}{l}5 /-5 \\
18-22 \\
36-41\end{array}$ & & $19-20 ; 24-25$ \\
\hline $\begin{array}{l}\text { Cat } 13 \\
\text { (vm LP1-c) }\end{array}$ & $\begin{array}{l}5 /+20 \\
14-16\end{array}$ & & $\begin{array}{l}0 /+5 \\
13-21\end{array}$ & $\begin{array}{l}0 /+2.5 \\
13-23\end{array}$ & $\begin{array}{l}0 / 0 \\
17-23\end{array}$ & $\begin{array}{l}5 /-5 \\
17-23\end{array}$ & $\begin{array}{l}0 /-2.5 \\
13-21\end{array}$ & & $\begin{array}{l}10 /-10 \\
16-25\end{array}$ & & $\begin{array}{l}18-23 ; 28-29 \\
35-38 ; 41-42\end{array}$ \\
\hline $\begin{array}{l}\text { Cat } 14 \\
\text { (dl LP1-c) }\end{array}$ & $\begin{array}{l}20 /+5 \\
19-22 \\
40-42\end{array}$ & & $\begin{array}{l}5 /+5 \\
15-21 \\
42-43\end{array}$ & $\begin{array}{l}0 /+2.5 \\
15-24\end{array}$ & $\begin{array}{l}0 /-1 \\
16-23 \\
33-35\end{array}$ & $\begin{array}{l}10 /-5 \\
22-24\end{array}$ & & $\begin{array}{l}5 /-7 \\
18-23\end{array}$ & $\begin{array}{l}10 /-15 \\
19-27 \\
31-32\end{array}$ & & $23-25$ \\
\hline $\begin{array}{l}\text { Cat } 15 \\
\text { (dl LP1-c) }\end{array}$ & $\begin{array}{l}5 /+5 \\
17-20 \\
40-41\end{array}$ & & $\begin{array}{l}0 /+5 \\
17-20\end{array}$ & & $\begin{array}{l}0 / 0 \\
17-20\end{array}$ & & $\begin{array}{l}5 /-5 \\
17-22\end{array}$ & & & & \\
\hline $\begin{array}{l}\text { Cat } 16 \\
\text { (dl LP1-c) }\end{array}$ & $\begin{array}{l}40 /-5 \\
20-24 \\
38-40\end{array}$ & & $\begin{array}{l}0 /+2.5 \\
20-24 \\
31-41\end{array}$ & & & & $\begin{array}{l}5 /-5 \\
20-22\end{array}$ & $\begin{array}{l}0 /-10 \\
18-25 \\
33-39\end{array}$ & & & \\
\hline $\begin{array}{l}\text { Cat } 17 \\
\text { (vm LP1-c) }\end{array}$ & $\begin{array}{l}5 /-5 \\
13-18\end{array}$ & & & & & & $\begin{array}{l}5 /-5 \\
13-21\end{array}$ & & & $13-21$ & \\
\hline
\end{tabular}

Values are for azimuth/elevation $\left({ }^{\circ}\right)$ in the top row for each cat, beta band ( $\left.\mathrm{Hz}\right)$ in the middle row, and gamma band ( $\left.\mathrm{Hz}\right)$ in the bottom row; vm, ventromedial; dl, dorsoventral.

Table 2. Data from Bekisz and Wrobel (2003) used for the histogram presented in Figure 3D

\begin{tabular}{|c|c|c|c|}
\hline & \multicolumn{3}{|l|}{ VCx area 17} \\
\hline & \multicolumn{3}{|r|}{$\longrightarrow$ anterior } \\
\hline \multirow[t]{3}{*}{ Cat 1} & $0 /-1.5$ & $-0.5 /-5$ & $0 /-10$ \\
\hline & $12-19$ & $12-17$ & $11-21$ \\
\hline & & $31-32$ & \\
\hline \multirow[t]{3}{*}{ Cat 4} & $+2.5 /+3$ & $0 /+1.5$ & $+2 /-2$ \\
\hline & $12-19$ & $12-17$ & $11-20$ \\
\hline & & & $33-34$ \\
\hline \multirow[t]{3}{*}{ Cat 5} & $+0.5 /+2.5$ & $+2 / 0$ & $-1.5 /-2$ \\
\hline & $12-20$ & $12-18$ & $11-18$ \\
\hline & $31-32,38-40$ & & $38-39$ \\
\hline \multirow[t]{3}{*}{ Cat 6} & $0 /+1$ & $0 /-2$ & $-0.5 /-10$ \\
\hline & $16-17,21-24$, & $12-19,30-$ & $12-19,22-23$, \\
\hline & $30-31$ & $34,38-39$ & $32-34$ \\
\hline
\end{tabular}

Values are for azimuth/elevation $\left(^{\circ}\right)$ in the top row for each cat, beta band $(\mathrm{Hz})$ in the middle row, and gamma band $(\mathrm{Hz})$ in the bottom row.

vious one. Before Fourier transformation, the raw data within the epochs were multiplied by the Hanning window function. The mean spectra were always calculated separately for individual animals and individual recording sites. Means were obtained from the first 3-6 recording days by averaging unitary spectra for all data epochs (excluding epochs contaminated by movement artifacts) from all trials of a given modality that ended with the same (correct or incorrect) behavioral response. Significant differences between mean visual and auditory FFT amplitude values were calculated at each frequency with the Student's $t$ test. To reveal whether histograms of summed frequency bands were similar, we conducted the Kolmogorov-Smirnov (KS) test, comparing the distribution of the two samples. Dependencies between two factors were evaluated by Pearson correlation coefficient. Because no stimuli were present during the analyzed period, and cats could not anticipate the side of cue stimuli appearance, we did not make a distinction in the analysis of trials terminated by left and right cues.

For additional correlation analysis, LFPs were digitally bandpass filtered (100th-order Finite Impulse Response filter with Kaiser window and no phase shift) with half-amplitude cutoff frequencies of 12-25, $12-19$, and $17-25 \mathrm{~Hz}$. These frequency bands were chosen to encompass the whole range of $\beta$, low-frequency $\beta 1$, and high-frequency $\beta 2$ that were enhanced during the visual task at specific regions of the investigated visual structures (compare Figs. 3, 6). To measure phase correlations, the filtered LFPs were subjected to the Hilbert transform (Barlow, 1993) to obtain the instantaneous amplitude of the oscillatory signals. Then, the raw filtered activities were divided by their amplitude envelope and changed to oscillatory signals of constant amplitude equaling 1 . Phase-cross-correlation functions were calculated first for single trials (time lag varied from -1 to $1 \mathrm{~s}$ in steps of $2.5 \mathrm{~ms}$; correlated signals were always corrected for zero means) and then averaged over trials. The maximal correlation values were taken for comparisons. 
Running correlation was also performed on the signals with constant amplitude, using a rectangular $125 \mathrm{~ms}$ window, $2.5 \mathrm{~ms}$ step, and zero time lag between signals. The final mean values of running correlation analysis were calculated by grand averaging of all unitary results obtained from individual trials of a given modality that ended with a correct behavioral response (trials contaminated by movement artifacts were excluded) for all available electrode pairs located in any two visual centers under investigation. The difference between mean visual and auditory values was calculated using two-way ANOVA (factors: modality and electrodes; the indicated probability values correspond to the modality factor).

\section{Results}

We have analyzed the spectral content of LFPs recorded between the cue stimulus, which directed the animals' attention to either the visual or auditory modality, and the suitable target stimulus, which indicated the appropriate reward feeder. Data from 58 recording sites in the left hemisphere of 10 cats were analyzed: 11 in the caudal part of the lateral zone of the LP-P (LPl-c), 6 in the rostral part of the lateral zone of the LP-P (LPl-r) and pulvinar proper, 35 in visual cortical areas 17 and 18, and 6 in the MSS [often called the lateral suprasylvian visual region (LS)]. Among the latter, three were placed in the medial bank of the MSS, in areas PMLS and AMLS, and three in its lateral bank, in areas PLLS and ALLS (for specification of the recording sites in each cat, see Tables 1, 2; Fig. 5). In most cases, the electrodes implanted in both cortical projection areas were placed pair-wise in approximately similar retinotopic positions: within the representation of the area centralis and at $\sim 5^{\circ}$ eccentricity. Nine recording sites were located at more peripheral representations of the visual field ( $\sim 5-20^{\circ}$ of azimuth and -10 to -15 elevation angles). Additional data from our previous experiment (Bekisz and Wróbel, 2003) from 12 recording sites in area 17, obtained in four other cats (Table 2) were included to enlarge the sample in Figure $3 D$. From these animals, cat 6 was trained in the same paradigm as that used in the present experiment, but cats 1,4 , and 5 saw a small, moving light stimulus $\left(0.5 \times 1.0^{\circ}\right)$ during the anticipatory period of the trial. The distribution of data used for analysis from the two groups of cats (with and without the moving spot) were shown to be the same $(12-24 \mathrm{~Hz} ; p=0.65 ; \mathrm{KS}$ test for comparing distributions).

\section{Cortical recordings}

In accordance with our previous experiments (Bekisz and Wróbel, 1993, 2003; Wróbel et al., 1994), anticipation of visual but not auditory target stimuli increased the number and amplitude of short (100-350 ms) bursts of oscillations in the $\beta$-frequency range $(12-29 \mathrm{~Hz})$ recorded in both striate and extrastriate cortical areas (data not shown). Accordingly, the amplitude of the FFT spectra within the $\beta$-frequency band was significantly elevated (Fig. 2A,B). These elevations were consistently observed only in the visual trials that ended with correct responses and were not seen in recordings preceding incorrect choices for the food reward (example in Fig. $2 C$ ). Note that the enhanced $\beta$ activity could not be related to any specific perceptual mechanism because no visual stimulus was present during several seconds of the analyzed anticipatory period of recording. The only difference between the visual and auditory conditions was the modalityspecific attentional activation started by cue stimulus within one of the two systems (Bekisz and Wróbel, 1993; Maunsell, 2004). Similar results were obtained for the first time from MSS recording sites (example in Fig. 2D). The frequency ranges of significant differences between FFTs obtained in visual and auditory trials from all cortical recording sites were compiled to show the dis-
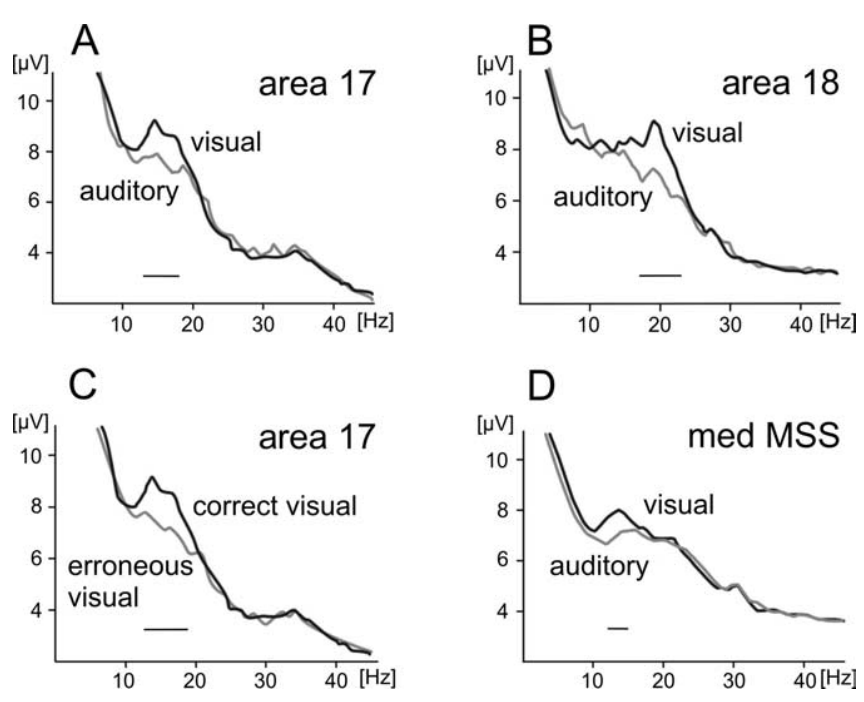

Figure 2. $\quad \beta$ Activity is increased during the anticipatory period of the trials. Mean FFT amplitude spectra for individual cortical sites in area 17 or 18 of cat 9 attentively expecting visual or auditory target stimuli. The analysis was done on signals recorded during the time interval between cessation of the cue and onset of the target stimuli. $A, B, D$, Averages were taken only from trials that ended with correct behavioral responses. The gray line in $C$ shows FFT obtained during visual trials that ended with an incorrect reward choice. Means were obtained from the first three recording days by averaging unitary spectra for all data epochs (1.28 s each; epochs with artifacts were excluded) during all trials of a given modality that ended with the same (correct or incorrect) behavioral responses (for details, see Materials and Methods). From "correct" trials, $\sim 600$ epochs were averaged for each site; for "incorrect" trials, 100 epochs were averaged. Black lines above the horizontal axis denote significantly different frequency ranges between the spectra (Student's $t$ test, $p<0.01$ ). Note that, in these examples, activity in areas 17 and PMLS was enhanced during visual trials in the lower $\beta$-frequency range $(\beta 1)$ but, at the same time, area 18 recordings exhibited larger amplitudes in higher $\beta$ frequencies $(\beta 2)$.

tribution of frequencies most activated by visual attention (Fig. $3 A, C, D)$.

Additionally, a number of recording sites available in the cortex showed that the ranges of elevated $\beta$ activity differed between cortical areas 17 and 18 (Tables 1,2). To better reveal these differences, we have included data gathered in our previous experiment that was devoted solely to $\beta$ activity in LGN and primary cortical sites (Bekisz and Wróbel, 2003) (Tables 1, 2). The LFPs obtained from area 17 (Figs. $2 A, 3 D$ ) showed averaged enhancement of activity in the whole $\beta$ range $(12-24 \mathrm{~Hz} ; 12-20 \mathrm{~Hz}$ at half-maximal value on the averaged band histogram) (Fig. $3 D$ ), whereas recordings in area 18 (Figs. $2 \mathrm{~B}, 3 \mathrm{C}$ ) showed an increase in the amplitude of the spectral content predominantly in higher $\beta$ frequencies (18-24 Hz at half-maximal value). The distributions obtained for area 17 and 18 were shown to be different as measured at the whole $\beta$ range (12-29 Hz; $p=0.047$; KS test). Note that this difference is probably not biased by the retinotopic position of available recording sites in areas 17 and 18 because the distributions calculated separately from recordings at central (eccentricity $<5^{\circ}$ ) and peripheral loci of both areas were similar ( $p=1.0$ to $p=0.15$; KS test).

The number of recording sites in different areas of the MSS was limited, and therefore, we pooled our data into two groups located in either the lateral or medial bank of MSS. The summed histogram calculated from recordings in the MSS covered the whole $\beta$ range (13-25 Hz at half-maximal value), and its distribution was similar to that obtained in areas 17 ( $p=0.84$, KS test) (Fig. $3 A, D)$ and $18(p=0.56)$ (Fig. $3 A, C)$. Note that, during visually attentive trials, FFT amplitudes recorded from sites located in the medial bank of the MSS increased predominantly in 

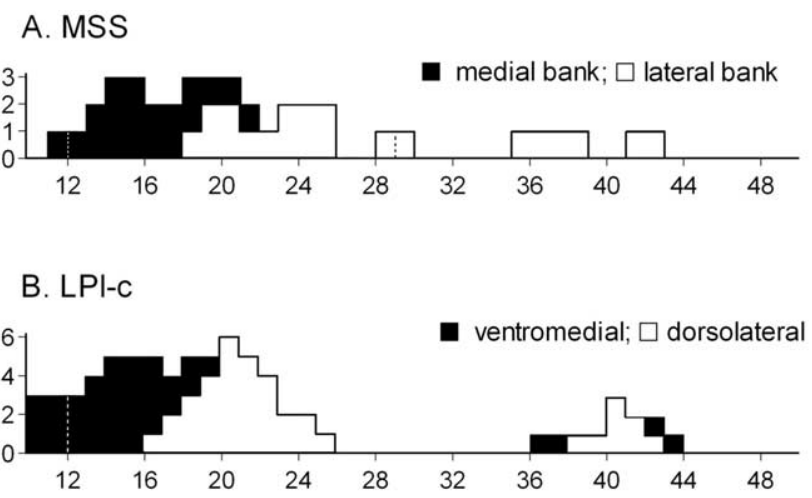

\section{VCx area 18}

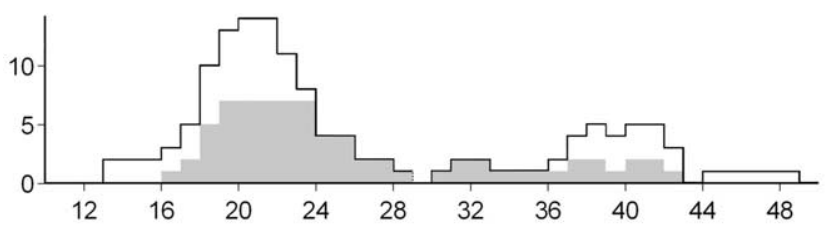

\section{VCx area 17}

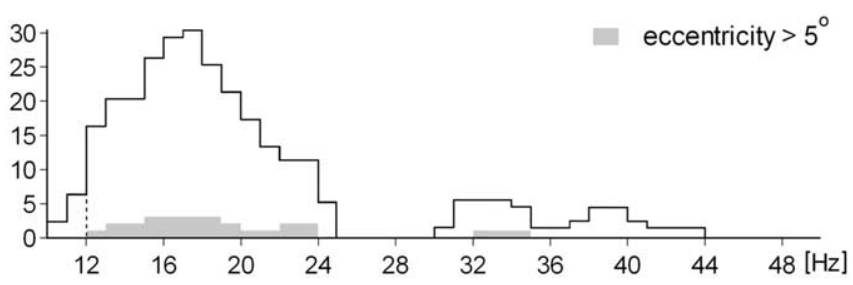

Figure 3. Frequency bands that were increased by attentive anticipation of the target stimuli. Histograms resulting from stacking together the frequency ranges with significant differences between FFTs obtained from visual and auditory attentive trials at all investigated levels of the visual system (see Results for details). $\boldsymbol{A}, \mathrm{MSS} ; \boldsymbol{B}, \mathrm{LPI}-\mathrm{C} ; \boldsymbol{C}$, area $18 ; \boldsymbol{D}$, area 17. Black parts in the two top histograms indicate stacked ranges of significantly different FFT amplitudes obtained from the ventromedial core region of $\mathrm{LPI}-\mathrm{c}(>0.8 \mathrm{~mm}$ from pulvinar border) and from medial bank of the MSS. White parts of these histograms represent the dorsolateral LPI-c and lateral bank of MSS, respectively. Each histogram consists of a different number of animals/recording sites: area 17 (14/30), area 18 (10/17), LPI-c (10/10), and MSS (6/6). For details, see Tables 1, 2.

the lower $\beta$ range (Fig. $3 A$ ) (13-18 $\mathrm{Hz}$ at half-maximal value), whereas recordings from the lateral bank of MSS showed an enhancement in the high $\beta$-frequency ranges (Fig. $3 A)(19-25 \mathrm{~Hz}$ at half-maximal value). Accordingly, MSS histograms have a clearly bimodal distribution, with peaks located in high and low $\beta$ frequencies formed by data from medial and lateral loci $(p<0.001$, KS) (Fig. 3A). Note, however, that histograms obtained from data in the medial MSS bank versus area 17 and the lateral MSS bank versus area 18 are alike ( $p=0.95$ and 0.27 , correspondingly, KS test) (Fig. $3 A, C, D$ ).

Thus, we found that $\beta$ activation during the attentive visual state also encompasses the MSS cortex in accordance with one of the driving pathways of visual processing (Sherman and Guillery, 2002). The available data suggests a causative link between $\beta$ activity and the known anatomical connections of MSS (Garey et al., 1991; Huppe-Gourgues et al., 2006), which could link $\beta$ activity between the primary visual cortex and MSS.

\section{LP-P recording sites}

According to the classification of Hutchins and Updyke (1988, 1989), the LP-P of the cat consists of functionally different zones,
A
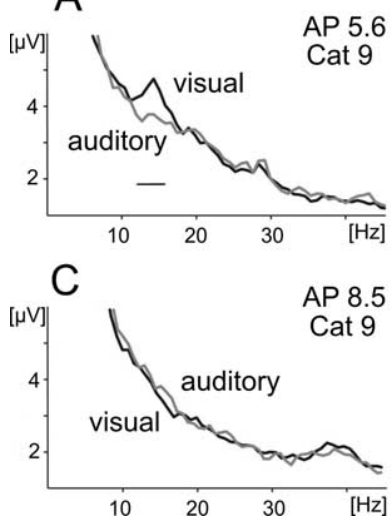

Figure 4. Increased $\beta$ activity during attentive anticipation of the target stimuli in LPI-C. $\boldsymbol{A}-\boldsymbol{D}$, Mean visual and auditory FFT amplitude spectra obtained for LFPs recorded from individual sites in different regions of lateral zone of the lateral posterior-pulvinar complex in cat $9(\boldsymbol{A}$, $\boldsymbol{C})$ and $10(\boldsymbol{B}, \boldsymbol{D})$. The analysis encompassed signals recorded during the time interval between cessation of the cue and onset of the target stimuli. $\boldsymbol{A}-\boldsymbol{C}$ contain exclusively those trials that ended with correct behavioral responses. The spectra are presented with reference to the location of the recording electrodes, i.e., from the most caudal $(\boldsymbol{A})$ through intermediate $(\boldsymbol{B})$ to the most rostral ( $C$ ) site according to Horsley-Clarke coordinates (compare the exact locations in Fig. 5). $\boldsymbol{A}-\boldsymbol{C}$, Different recording sites are located in the ventromedial core of $\mathrm{LPI}-\mathrm{C}(\boldsymbol{A})$, the dorsolateral region of $\mathrm{LPI}-\mathrm{C}(\boldsymbol{B})$, and the rostral part of $\mathrm{LPI}(\boldsymbol{C})$. D, FFT spectra averaged from visual trials ending with a correct response (black line) are compared with those ending with an incorrect behavioral response. Note the enhancement of low-frequency $(\beta 1)$ activity in the ventrolateral region of $\mathrm{LPI}-\mathrm{C}(\boldsymbol{A})$ and high-frequency $(\beta 2)$ activity in dorsolateral region of $\mathrm{LPI}-\mathrm{C}(\boldsymbol{B})$. No enhancement of $\beta$ activity was observed in LPI-r ( $C$ or during incorrectly ended visual trials in LPI-c (D; gray line). In each cat, $\sim 600$ data epochs from first three recording sessions were used to calculate mean spectrum for trials with correct response and 50 epochs were taken to obtain mean spectrum for trials with incorrect reward choice. Other explanations are as in Figure 2.

which have distinct connections with cortical areas and subcortical structures. One of them, the lateral zone (LPl), includes the LPl-c and LPl-r. The large caudal part is the region of LPl that receives strong "driving input" (Guillery et al., 2001) from and sends reciprocal connections to areas 17 and 18 and was therefore called the "striate-recipient zone" (Updyke, 1977; Raczkowski and Rosenquist, 1983). The cells in LPl-c and striate cortex exhibit many similar response properties, such as binocularity, orientation, and direction selectivity, but LPl-c neurons often possess larger and more complex receptive fields than those seen in the striate cortex (Harutiunian-Kozak et al., 1981; Chalupa and Abramson, 1989; Casanova et al., 1997). In addition, LPl-c is reciprocally interconnected with many other visual areas including those located in the suprasylvian sulcus (for review, see Garey et al., 1991). Accordingly, some of the complex functional properties of LPl-c neurons like binocular facilitation (Rauschecker et al., 1987) and global motion processing (Rudolph and Pasternak, 1996; Merabet et al., 2000; Dumbrava et al., 2001) were attributed to the inputs from the "lateral syprasylvian cortex" located at the banks of the middle suprasylvian sulcus (Grant and Hilgetag, 2005). The LPl-r receives its predominant input from the midbrain visual centers, instead of projections from striate cortex, and is reciprocally interconnected with a different set of cortical visual areas (Garey et al., 1991).

To define the functional role of the lateral posterior-pulvinar complex in visual processing, we analyzed attention-related changes in the FFT amplitude spectra of local field potentials recorded from the three main divisions of this complex (LPl-c, LPl-r, and pulvinar proper). The examples of averaged FFT spectra calculated from different recording sites in the LP-P complex during visual and auditory trials are shown in Figure $4 A-C$. 


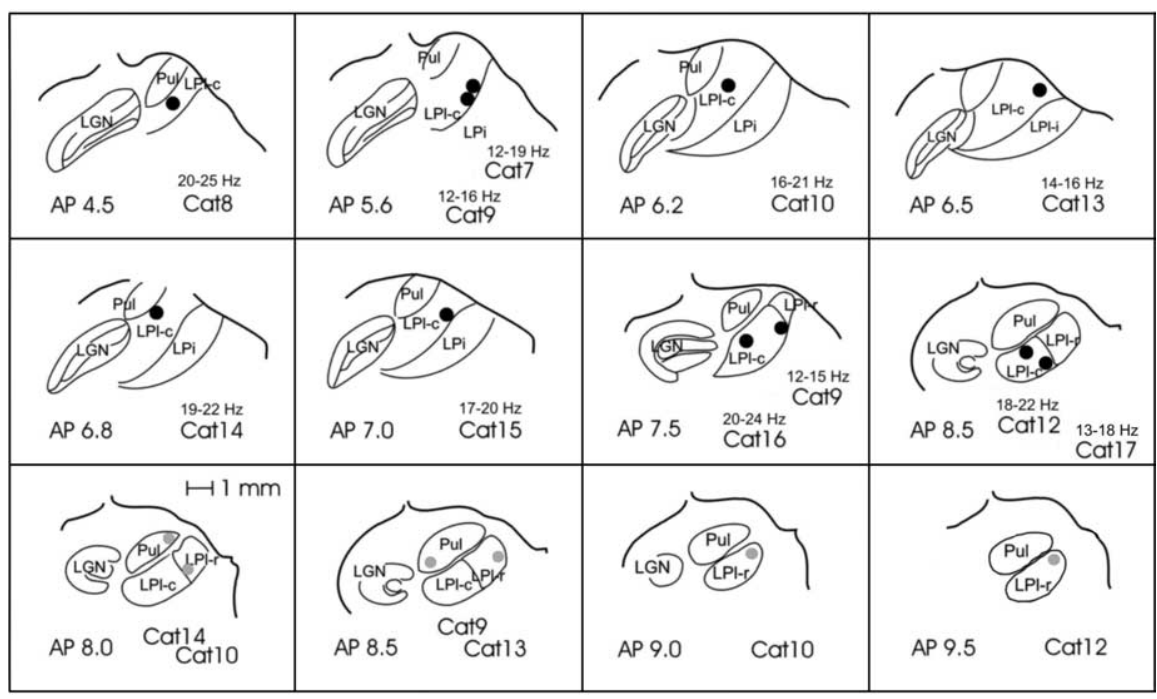

Figure 5. Schematic drawing showing all of the recording sites in LP-P. The two top rows show locations where $\beta$-activity enhancement during visual trials was statistically significant (ranges are given above the cat number). Sections are ordered from most posterior (top left) to most anterior (middle row, right) according to Horsley-Clarke coordinates. The third row shows the locations where no increase of $\beta$ activity was recorded. Pul, Pulvinar zone of LP-P; LGN, lateral geniculate nucleus. $\beta$ range (12-25 Hz) (Fig. 3B). This increase was similar to that found in area $17(p=$ 1.00 , KS test for similarity of distributions), area $18(p=0.26)$, and MSS ( $p=$ $0.97)$. The histological verification of recording sites (Fig. 5) revealed that the frequency range activated by increased visual attention was dependent on the recording location within this region. We found that the recordings obtained from sites located not further than $0.8 \mathrm{~mm}$ from the pulvinar border, in the dorsolateral segment of the LPl-c, showed an increase of visual attention-related activity within the upper $\beta$ range (16-25 Hz) (Fig. $4 B$; compare recording locations in Figs. 5, 6), whereas activity in ventromedial loci showed an increase within the lower $\beta$ range (12-19 $\mathrm{Hz}$ ) (see Figs. 4A, 5, 6). The distributions of these attention-sensitive frequency ranges from both LPl-c regions were clearly different $(p<0.001$, KS test). Furthermore, the distribution of attentioninfluenced frequencies in the dorsolateral

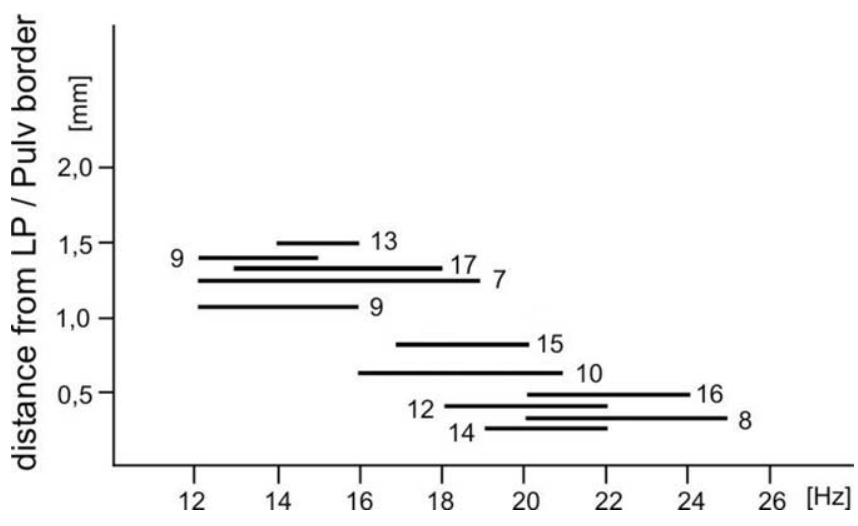

Figure 6. Frequency ranges of significant differences between FFTs obtained from all recording sites in LPI-c. The LFP signals taken for analysis were recorded during correctly ended visual and auditory trials. Ordinate indicates shortest distance (in three-dimensional space) between the electrode location and the pulvinar border. Numbers specify experimental animals. Pulv, Pulvinar zone of LP-P.

These examples were obtained from two animals and are presented with reference to the location of the recording electrodes, i.e., from the most caudal (Fig. 4A) through intermediate (Fig. $4 B$ ) to the most rostral (Fig. $4 C$ ) site according to Horsley-Clarke coordinates. The elevated $\beta$ activity observed during visual trials was only seen in the recording sites located in LPl-c (Fig. 4A, B) and not in pulvinar proper (Fig. $4 C$ ). Similar to the cortical recordings, the $\beta$-frequency enhancement in LPl-c accompanied only trials that ended with a correct behavioral response (Fig. $4 D$ ), thus providing additional evidence for its involvement in an attentional mechanism. The locations of all recording sites in LP-P, together with the frequency ranges of significant $\beta$ enhancement during visual trials are presented in Figure 5. Data from the lowermost row of this figure show that, in contrast to LPl-c (the striate-recipient zone), $\beta$ power was not enhanced during visual trials either in the rostral part of LPl nor in the pulvinar proper.

The distribution of significant $\beta$ activity increases during attentive visual trials recorded from LPl-c encompassed the whole segment (Fig. 3B, white area of the histogram) was also different from that in area 17 ( $p=0.02$, KS test) (Fig. 3D) but matched the distribution obtained in area $18(p=1.00)$ (Fig. $3 C)$. At the same time, the distribution found in the ventromedial part of LPl-c (Fig. 3B, dark area of histogram) was similar to that found in area $17(p=0.17)($ Fig. $3 D)$ and differed from the area 18 distribution $(p<0.001)$ (Fig. 3C).

Note that the division of LPl-c into dorsolateral and ventromedial regions was unexpected and based solely on physiological data that grouped the recordings sites with high and low $\beta$-frequency activations according to their distance from the pulvinar border (Fig. 6). Although the number of our recording sites in LP1-c is limited (10 in 11 cats), the proposed division perfectly matches the diverse morphology and connectivity of these subregions (Berson and Graybiel, 1978; Updyke, 1983; Abramson and Chalupa, 1988; Garey et al., 1991; Kelly et al., 2003; Huppe-Gourgues et al., 2006) and differences of their neuronal responses in anesthetized cats (Casanova et al., 1989; Chalupa and Abramson, 1989; Hutchins and Updyke, 1989; Dumbrava et al., 2001). There is, however, a discrepancy between our data and those of others with regard to the extent of the dorsolateral region bordering on the pulvinar. Previous data (Chalupa and Abramson, 1989) estimated this distance to be $\sim 0.4 \mathrm{~mm}$ along the vertical penetration of the recording electrode, whereas our histological reconstructions of electrode locations at which recording of higher-frequency $\beta$ activity occurred were localized as far as $0.7 \mathrm{~mm}$ from the $\mathrm{LPl} / \mathrm{P}$ border (Fig. 6). We attribute this difference to the large volume of tissue contributing to LFPs that originate from signal sources located up to $0.4 \mathrm{~mm}$ from the electrode tip and may cause such error.

\section{Increased gamma activity associated with visually attentive situations}

Finally, it should be noted that, in agreement with our previous findings (Bekisz and Wróbel, 1999), we frequently noticed elevated activity in the gamma band in both visual and auditory situations. For a number of visual trials, gamma activity increased in parallel with $\beta$ attentional modulation reaching statistical sig- 


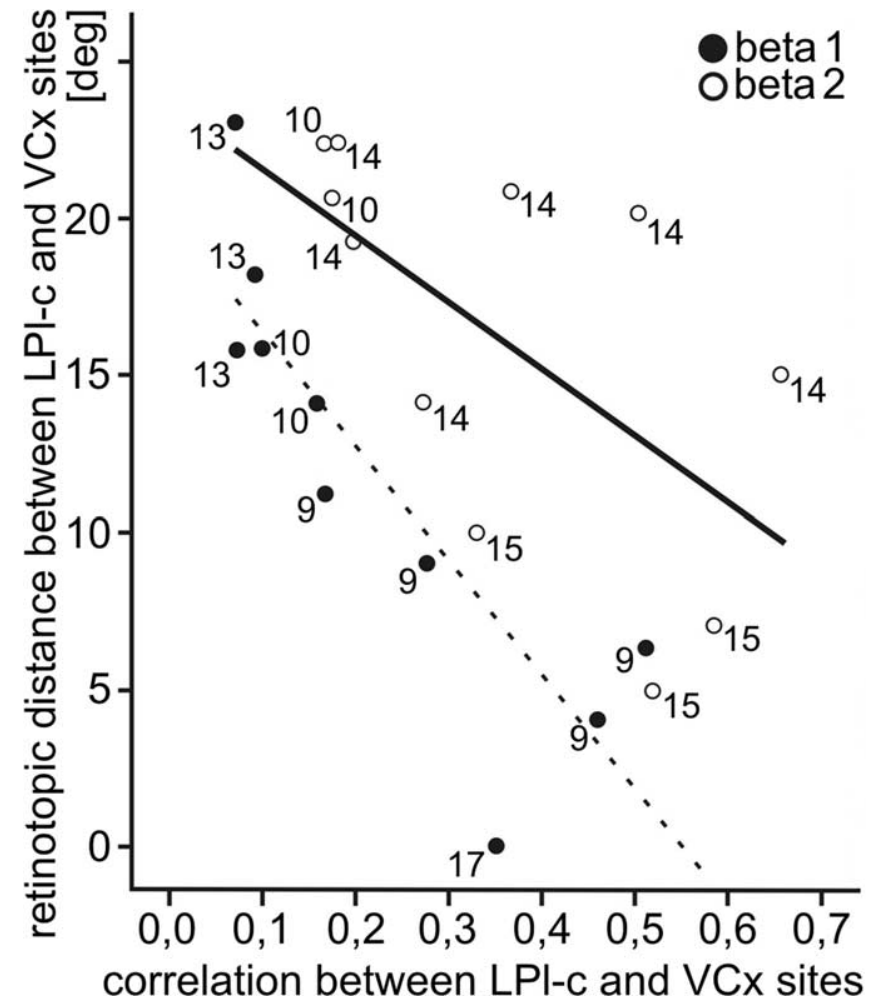

Figure 7. $\beta$ Synchronization depends on the retinotopic distance between cortical and LPI-C recording sites. Phase-correlation coefficients calculated for the sample of corticothalamic pairs of LFP signals with matched ranges of increased attentional $\beta$ activity plotted against distance between the retinotopic positions of cortical and thalamic sites are shown. The recordings from these pairs were filtered at $\beta 1(12-19 \mathrm{~Hz})$ or $\beta 2(17-25 \mathrm{~Hz})$ ranges. The two first seconds of recording after the presentation of the visual cue were taken for analysis. The graph shows correlation values averaged over visual trials from three to six experimental days. Filled circles indicate pairs of recordings from $\mathrm{LPI}$-c and cortical sites that were characterized by the increased attention-related visual activity within the low-frequency $(\beta 1)$ range. Open circles denote pairs of recordings showing increases in the high-frequency $(\beta 2)$ range. Numbers indicate cats from which correlation values were calculated. Data recorded from cats 7, 12, and 16 were not included because of recording artifacts that did not allow for correlation analysis over a long period of time. Pearson's correlation for the whole sample, -0.509 ( $p=0.018)$; within $\beta 1$ pairs, -0.851 ( $p=0.002)$; and within $\beta 2$ pairs, -0.603 ( $p=0.05)$.

nificance in all investigated visual regions (Fig. 3, frequencies $>30 \mathrm{~Hz}$ ).

\section{Correlations of $\boldsymbol{\beta}$ activity between cortical and thalamic recordings}

To determine whether the dorsolateral and ventromedial regions of the LPl-c, which expressed enhanced $\beta$ activity of different frequencies in visually attentive situations, would also exhibit different functional connections with cortical areas 17 and 18, we correlated LFPs recorded from all of the available corticothalamic pair sites. In line with our previous study on geniculocortical loops (Bekisz and Wróbel, 2003), we have not found the attention-dependent change in correlations calculated between LPl-c and primary cortex signals with either raw or envelope techniques. This was not surprising, because the two signal samples (from LPl-c and cortex) represented clearly different, central and peripheral retinotopic locations, and attention-dependent coupling between envelopes of $\beta$ activity was previously confirmed only for these cortical and geniculate recording sites that both represented the central visual field (Bekisz and Wróbel, 2003). In contrast, neurons in the LPl-c have larger receptive fields and may thus detect weak signals from distant sources. We
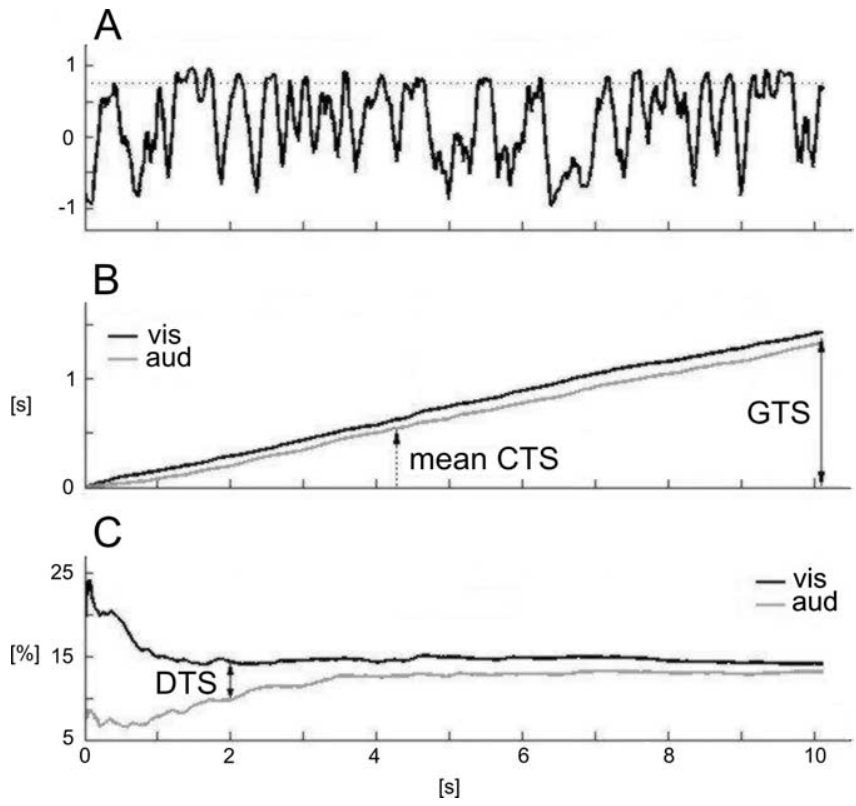

Figure 8. An example of evaluating the DTS between two LFP signals. $\boldsymbol{A}$, Running phase correlation calculated between 12 and $25 \mathrm{~Hz} \beta$ signals recorded during a single visual trial from two electrodes located in $\mathrm{LPI}-\mathrm{c}$ and area 17 in cat 9 . The time epochs during which the phase correlation exceeded 0.75 were continuously summed starting from the offset of the cue stimulus and formed a cumulated function for each experimental trial. $\boldsymbol{B}$, The cumulated functions obtained from 38 visual (vis) and 45 auditory (aud) trials recorded in three consecutive daily sessions were averaged to measure CTS during visual and auditory trials. $\boldsymbol{C}$, The relative time of high synchronicity as measured from the offset of the cue stimulus in relation to time of the trial $(\mathrm{CTS} \times 100 \% / \mathrm{t})$. The mean percentage DTS between visual and auditory trials was measured at $2 \mathrm{~s}$ after cue stimulus offset and compared between all recording sites as shown in Figure 9.

have therefore checked whether the temporary state of congruent activity at LPl-c and cortical sites would be expressed by phase correlation, which does not take into account the amplitude of synchronous oscillations (Varela et al., 2001) (for details, see Materials and Methods). We found that the correlation coefficients calculated between the signals that expressed enhanced $\beta$ activity at the same subrange (low or high) were bigger than those between recordings with mixed functional $\beta$ subranges that never exceeded 0.25 (data not shown). Figure 7 shows the mean phasecorrelation coefficients for all available pairs that expressed matched ranges of enhanced $\beta$ activity during visual trials. They were calculated within low $(12-19 \mathrm{~Hz})$ or high $(17-25 \mathrm{~Hz})$ $\beta$-frequency windows for pairs with corresponding frequency ranges from data registered during the first $2 \mathrm{~s}$ of the anticipatory period in all experimental trials. Most of the obtained correlation coefficients were small, which might be partly caused by the fact that the investigated corticothalamic pairs did not match retinotopically (Table 1). Indeed, the larger the distance between retinotopic representations of the cortical and thalamic recording sites, the lower the correlation coefficient $(r=-0.509 ; p=$ 0.018) (Fig. 7).

To investigate the corticothalamic synchronization in more detail, we have calculated running phase correlations between all pairs of signals (example from one pair in Fig. $8 \mathrm{~A}$ ). Such a function continuously fluctuated and randomly reached high values even for those signal pairs that had small mean correlation values. It is often assumed that correlation values $>0.75$ might indicate a significant functional link (called below the high synchronicity) between the oscillating sites, as a square of this value exceeds 0.5 (Armitage and Berry, 1994; Wróbel, 2000). Accordingly, we have measured a cumulative time of high synchronicity (CTS) during 


\section{LPI-c vs VCx}
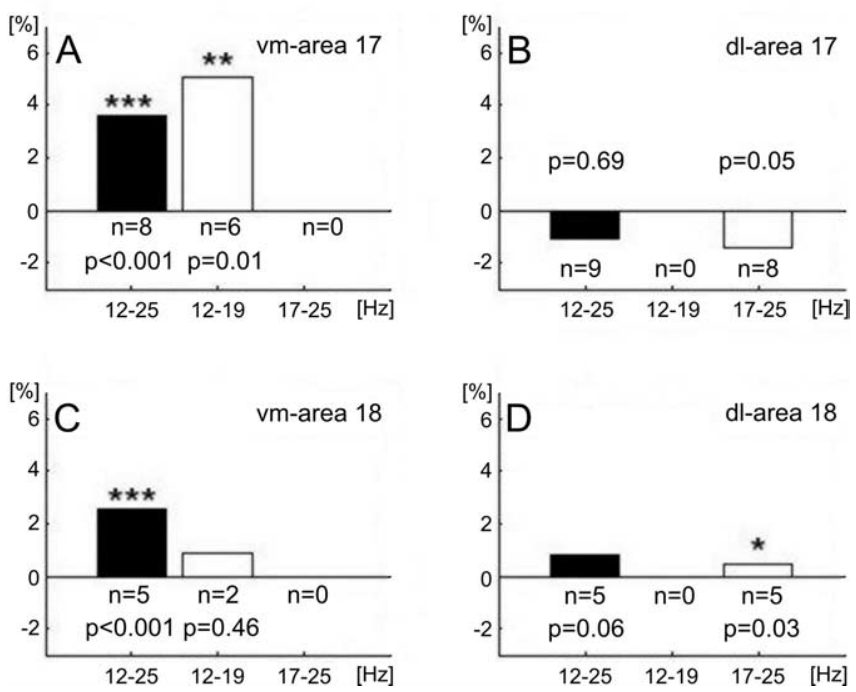

Figure 9. Synchronicity between attentional $\beta$ signals recorded from primary visual areas and LPI-C. Mean DTS values calculated for all connections between the visual cortex (areas 17 and 18) and two LPI-c regions (dorsolateral and ventromedial) are denoted by filled bars. Open bars indicate DTS values obtained between only those pairs of recordings that expressed matched $\beta$-frequency bands enhanced by the visual task (compare Tables 1, 2). The DTS values represent relative time of high thalamocortical synchronicity $(>0.75)$ during visual trials minus relative time of thalamocortical high synchronicity during auditory trials and is measured at $2 \mathrm{~s}$ recording time after cessation of the cue stimulus (see Fig. 8 for additional explanations). $\mathbf{A}-\mathbf{D}$, Signal frequency bands from which DTS values were calculated are shown below each box. Each of the mean DTSs represent grand averaging across trials and all available electrode pairs for the connection type indicated in the top right corner of the appropriate graph (e.g., vm-area 17). The probability values over bars indicate significant differences between visual and auditory relative times of high synchronicity measured at $2 \mathrm{~s}$ (two-way ANOVA, with factors: modality and electrodes; the indicated probability values correspond to the modality factor). vm, Ventromedial part of LPI-c; dl, dorsolateral part of LPI-c; $n$, number of pairs for each bar.

which a running correlation of $\beta$ signals $(12-25 \mathrm{~Hz})$ exceeded 0.75 , separately for visual and auditory trials (Fig. $8 B$ ). The global time of high synchronicity (GTS) between most $\beta$ signal pairs occupied on average $10-20 \%$ of the experimental trials and was typically longer for visual ones (Fig. $8 \mathrm{~B}$ ). The relative time of high synchronicity, as measured from the offset of cue stimulus in relation to the time of the trial (CTS/t), is exemplified in Figure $8 C$ for visual and auditory signals recorded in cat 9 . As in the data from other cats, the biggest difference between the mean relative times of high synchronicity calculated for visual and auditory signals was observed during the first $2 \mathrm{~s}$ of the trials and did not change for the remaining eight seconds (cf. Gross et al., 2004). Consequently, to evaluate the difference between the visual and auditory mean relative times of high synchronicity (DTS) for the whole sample, we measured this value at $2 \mathrm{~s}$ of each trial, as indicated in Figure 8C.

As shown in Figure 9, the significant positive mean DTS values were obtained in the full $\beta$ range $(12-25 \mathrm{~Hz})$ for correlations between both cortical primary areas and the ventromedial region of LPl-c (Fig. 9A, $C$, filled bars). This indicates that during the first $2 \mathrm{~s}$ of the visual trials, $\beta$ signals recorded from ventromedial LPl-c region and cortical primary areas synchronize more than during auditory trials. The more detailed analysis was performed on recording pairs that expressed enhanced $\beta$ activity during visual trials at the same $\beta$ subrange (low or high). For these sites, we performed correlation analysis using signals filtered specifically in the relevant (lower or higher) $\beta$-frequency range. The med MSS vs LPI-c
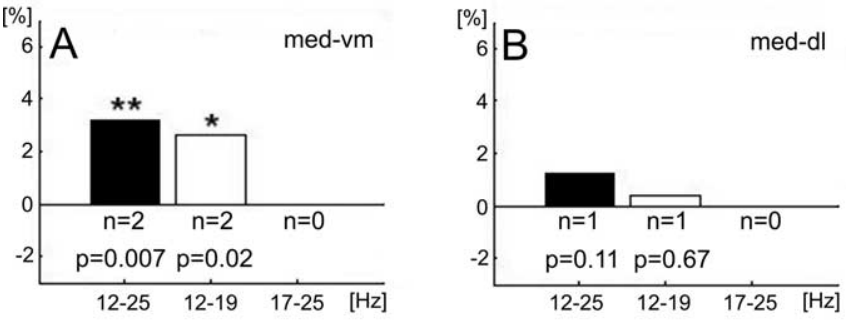

\section{med MSS vs VCx}
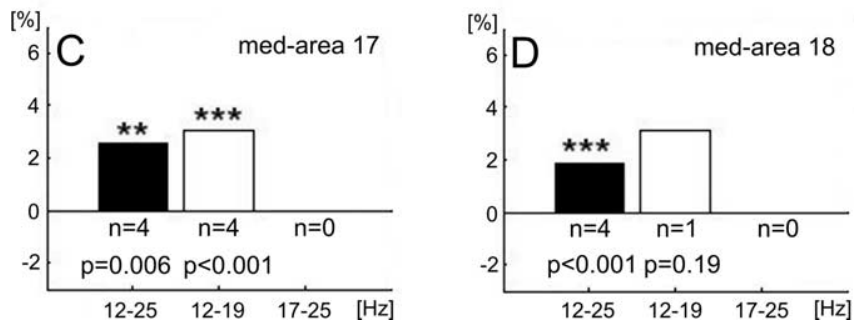

Figure 10. Synchronicity between attentional $\beta$ signals recorded from the medial MSS (med MSS) and other visual structures. DTS values showing high synchronicity of LFPs recorded simultaneously from sites in the medial bank of MSS and LPI-c or primary visual cortical areas. DTSs were calculated from signals filtered in full $\beta$ band $(12-25 \mathrm{~Hz}), \beta 1(12-19 \mathrm{~Hz})$, and $\beta 2$ $(17-25 \mathrm{~Hz})$. Note that synchronicity between the ventromedial part of the LPI-c and the medial MSS and between area 17 and medial MSS was enhanced during attentive visual behavior predominantly in the low-frequency $(\beta 1)$ band. Significance values were calculated with ANOVA. Other explanations are as in Figure 9.

DTS values calculated from these data show that area 17 synchronized its activity in the low $\beta$-frequency range only with the ventromedial region of LPl-c ( $p=0.009)$ (Fig. 9A), whereas area 18 synchronized with dorsolateral LPl-c in high $\beta$ range $(p=0.03)$ (Fig. 9D, open bars). These results suggest that, during visual trials, two separate corticothalamic systems are synchronized by $\beta$ activity of different frequency ranges. The ANOVA analysis (see Materials and Methods) has additionally shown an effect of interaction between modality and electrode factors. This effect suggests that recordings from different pairs of electrodes were synchronized at different levels, consistent with the results presented in Figure 7.

Similar calculations were performed for connections between recording sites located in the LPl-c and MSS and between primary areas and MSS cortical loci (Fig. 10). We found that signals from the medial bank of the MSS synchronized their visually related activity in the low $\beta$ range with the ventromedial LPl-c $(p=0.02)$ (Fig. 10A) and with area $17(p<0.001)$ (Fig. 10C). No significant correlations were found between signals from lateral MSS bank and primary cortical or thalamic recording sites (data not shown). Together, the correlation analysis supports our main finding that attention-dependent $\beta$ activities of particular $\beta$-frequency ranges characterize specific recording sites in the visual processing pathways (Figs. 3, 9, 10).

\section{Discussion}

In this study, we have shown that LFP recordings from the caudal part of the lateral zone of LP-P and the visual areas located in the middle suprasylvian sulcus of the cat exhibit increased $\beta$ (12-25 $\mathrm{Hz}$ ) activity during attention-related behavior. Such correlation, together with data from our previous investigations on the LGN and primary cortex (Bekisz and Wróbel, 1993, 2003; Wróbel et 

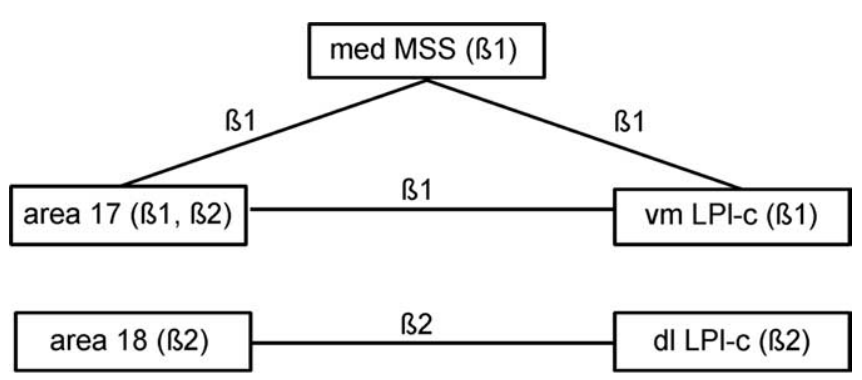

lat MSS (ß2)

Figure 11. Two streams of $\beta$ activity in the corticothalamic system of the cat. Our results suggest that during anticipatory visual attention two separate corticothalamic systems are activated and synchronized by different frequency bands of $\beta$ activity: $\beta 1(12-19 \mathrm{~Hz})$ and $\beta 2$ (17-25 Hz). vm LPI-c, Ventromedial part of LPI-c; dl LPI-c, dorsolateral part of LPI-c; med MSS, medial bank of middle suprasylvian sulcus. For anatomical locations of ventromedial and dorsolateral parts of LPI-c, see Figure 5.

al., 1994) indicate that these structures belong to the part of the visual system that was proposed to be set into attentional mode by the spread of $\beta$-oscillatory bursts via modulatory branches of involved loops (Wróbel, 2000). Second, our results indicate that, depending on the location in cortical areas $17 / 18$ and specific LPl-c and MSS subregions, $\beta$ activity in these centers occurs at two different frequency bands: $12-19 \mathrm{~Hz}$ and $17-25 \mathrm{~Hz}$, known as $\beta 1$ and $\beta 2$, respectively (von Stein et al., 1999). Consequently, the phase-correlation analysis showed that, during visually attentive situations, area 17 increases a functional link with the ventromedial region of the LPl-c and medial bank of MSS (at $\beta 1$ range), whereas area 18 synchronizes with the dorsolateral region of the LPl-c (at $\beta 2$ range). Our main findings are summarized in Figure 11.

\section{Relationship of attention-dependent $\boldsymbol{\beta}$ activity to anatomical and physiological studies}

The idea that the extrageniculate visual thalamus plays an important role in corticocortical communication is mainly supported by anatomical connectivity studies (for review, see Guillery and Sherman, 2002; Shipp, 2003). In line with well described, distinct anatomical connections, we have observed visual attentionrelated $\beta$ activity only in the striate recipient caudal part of the lateral zone ( $\mathrm{LPl}-\mathrm{c})$ and not in its tecto-recipient rostral part (LPl-r) nor in the pulvinar proper, which is mainly interconnected with higher visual areas and receives additional input from pretectal centers (Garey et al., 1991). It is worthy to mention, however, that our paradigm does not evoke stimulus driven attention. It remains to be verified whether attended moving stimuli would not increase $\beta$ activity in the LPl-r subregion of the LP-P.

Together with our previous findings in the LGN and striate cortex (Bekisz and Wróbel, 1993, 2003; Wróbel et al., 1994), the present data indicate that visual attention coincides with increased $\beta$ activity at consecutive stages of the visual processing pathway. In the anticipatory situation of the paradigm used in the present experiment, this activation could only be related to the general activation of the visual channel and not to the detailed analysis of the complex visual scene. This may explain why we have not observed a similar increase of $\beta$ activity in the pulvinar, because this zone of the cat's LP-P is putatively involved in higher-order visual processing (Chalupa, 1991; Sherman and Guillery, 2002).
Our correlation results suggest that activity of high and low $\beta$ frequencies might synchronize within two separate circuits interconnecting the cortical area 17 with ventromedial and area18 with dorsolateral part of striate recipient zone of LP-P (Fig. 11). It is well known that area 18 receives predominantly Y channel input (Stone and Dreher, 1973; Stone, 1983). This might suggest that the higher-frequency, $\beta 2$ stream would involve fast, Y-channel information. In accordance with this hypothesis, our recordings obtained during the visually attentive task showed parallel and phase correlated increases of high-frequency $\beta$ activity in area 18 and dorsolateral LPl-c (Figs. 3, 8). In this respect it might be important that the latter subregion also receives an additional input from the superior colliculus (Abramson and Chalupa, 1988). Whether the two $\beta$ frequencies are indeed spread by different visual pathways remains to be proven by further detailed analysis.

\section{Separate streams of different $\boldsymbol{\beta}$ frequencies in the visual system: functional considerations}

Our data suggest that during attentive behavior the striate recipient zone of the LP-P complex ( $\mathrm{LPl}-\mathrm{c}$ ) is segregated into dorsolateral and ventromedial regions by different frequency bands of $\beta$ oscillatory activity. The two functionally distinct regions of LPl-c may thus represent integrative relays for separate corticothalamocortical streams of information processing, dealing with different aspects of visual stimuli. This proposal is strongly supported by previous studies of anatomical connections (Berson and Graybiel, 1978; Updyke, 1983; Abramson and Chalupa, 1988; Kelly et al., 2003; Huppe-Gourgues et al., 2006) and single cell recordings from anesthetized cats (Casanova et al., 1989; Chalupa and Abramson, 1989; Dumbrava et al., 2001). The hypothesis proposed here is based on our clear-cut statistical analysis which matched two different bands of increased $\beta$ attentional activity with anatomically distinct locations at three levels of visual information processing (primary cortices, LPl-c and MSS) and additionally confirmed by correlation data in the functioning network. One of these corticothalamocortical streams would involve visual cortical areas (mainly area 18), the dorsolateral part of LPl-c and possibly the lateral bank of the middle suprasylvian sulcus which all displayed increased attention-related activity at the $\beta 2$ range (Fig. 3). The link between area 18 and dorsolateral LPl-c was also shown to synchronize at a high $\beta$ range during visually attentive trials (Fig. 9D). The other stream would encompass area 17, the ventromedial region of $\mathrm{LPl}-\mathrm{c}$, and the medial areas of MSS, which were activated mainly in the $\beta 1$ frequencies (Fig. 3), and increase their mutual synchronicity also at the same $\beta 1$ range during visually attentive situations (Figs. 9A, 10C,D, 11). Thus, our observations suggest that separate streams of attention-related corticothalamocortical $\beta$ activity involve different regions in LPl-c and may form thalamic counterparts for two processing pathways in the cortex.

Somewhat similar functional corticothalamocortical units have been previously proposed on the basis of purely anatomical (Symonds et al., 1981; Raczkowski and Rosenquist, 1983) or physiological (Dumbrava et al., 2001) results. The overall picture of the functional organization of the cat's LPl-c elaborated here would be remarkably similar to that for the monkey pulvinar complex recently proposed by Shipp (2004) on the basis of the anatomical connections and retinotopic mapping. He has proposed that the primate pulvinar complex, regardless of internal anatomical boundaries, might be divided into two functional parts, which are used by separate corticothalamocortical loops 
matching reciprocally ventral and dorsal pathways of cortical processing of visual information.

A recent viewpoint posits that the pulvinar complex of cats and monkeys acts as a link in corticocortical communication subserving its important role in situations requiring visual attention (Olshausen et al., 1993; Portas et al., 1998; Sherman and Guillery, 2002; Shipp, 2004). Our results provide the first direct, functional data suggesting a putative mechanism of such modulation, which is related to the spread of $\beta$ activity within the system.

Mounting evidence supports our hypothesis (Bekisz and Wróbel, 1993; Wróbel, 2000) that an increase in $\beta$-band activity plays an important role in attentional modulation. In behaving cats and monkeys, Rougeul-Buser and colleagues have recorded $20 \mathrm{~Hz}(\mu)$ activity in parietal cortex, during the stimulus expectancy period (Montaron et al., 1979), and they also showed similar rhythms preceding eye movements in behaving cats (Buser and Rougeul-Buser, 2005). The modulation in the $\beta$ band was also attributed to cognitive processes in cats (Liang et al., 2002), reported during perception-related binocular rivalry in monkeys (Gail et al., 2004) and object processing in men (von Stein et al., 1999). Interestingly, a few neuronal pairs recorded in the monkey extrastriate cortex were shown to synchronize at $\beta$ frequencies during attentive expectation (Cardoso de Oliveira et al., 1997). Recent magnetoencephalographic recordings in human subjects showed phase synchronization in the $\beta$ range along long-range attentional circuits including dorsal and ventral streams (Gross et al., 2004) and in intracranial EEG signals between a number of extrastriate areas during maintenance of objects in short-term memory (Tallon-Baudry et al., 2001). Finally, simulation studies indicated that $\beta$ oscillations might be favored during long-range interactions of widely distributed neuronal networks in contrast to gamma oscillations, which appeared to be more adequate for local synchrony (Kopell et al., 2000; Bibbig et al., 2002; Gail et al., 2004). These theoretical expectations are in line with our proposal that LFP $\beta$ bursts may be used for subthreshold activation of a corticogeniculate network subserving local gamma binding (Bekisz and Wróbel, 1999; Wróbel, 2000). Such a mechanism could be easily generalized for other recurrent loops. Indeed, multiunit activity in the cat's LP-P was shown to oscillate in the $\beta$ band during visual stimulation (Shumikhina and Molotchnikoff, 1995) and could therefore set the membrane potential of neurons in visual cortex (Llinas et al., 1991) to the level required for gamma oscillations and synchrony (Shumikhina and Molotchnikoff, 1999; Niebur et al., 2002).

\section{References}

Abramson BP, Chalupa LM (1985) The laminar distribution of cortical connections with the tecto- and cortico-recipient zones in the cat's lateral posterior nucleus. Neuroscience 25:81-95.

Abramson BP, Chalupa LM (1988) Multiple pathways from the superior colliculus to the extrageniculate visual thalamus of the cat. J Comp Neurol 271:397-418.

Armitage P, Berry G (1994) Statistical methods in medical research, Ed 3. Oxford: Blackwell Scientific.

Barlow JS (1993) The electroencephalogram: its patterns and origins. Cambridge, MA: MIT.

Bekisz M, Wróbel A (1993) $20 \mathrm{~Hz}$ rhythm of activity in visual system of perceiving cat. Acta Neurobiol Exp (Wars) 53:175-182.

Bekisz M, Wróbel A (1999) Coupling of beta and gamma activity in corticothalamic system of cats attending to visual stimuli. NeuroReport 10:3589-3594.

Bekisz M, Wróbel A (2003) Attention-dependent coupling between beta activities recorded in the cat's thalamic and cortical representations of the central visual field. Eur J Neurosci 17:421-426.
Bender DB, Youakim M (2001) Effect of attentive fixation in macaque thalamus and cortex. J Neurophysiol 85:219-234.

Berson DM, Graybiel AM (1978) Parallel thalamic zones in the LP-pulvinar complex of the cat identified by their afferent and efferent connections. Brain Res 147:139-148.

Berson DM, Graybiel AM (1983) Organization of the striate-recipient zone of the cats lateralis posterior-pulvinar complex and its relations with the geniculostriate system. Neuroscience 9:337-372.

Bibbig A, Traub RD, Whittington MA (2002) Long-range synchronization of gamma and beta oscillations and the plasticity of excitatory and inhibitory synapses: a network model. J Neurophysiol 88:1634-1654.

Buser P, Rougeul-Buser A (2005) Visual attention shifts and sustained attention episodes are accompanied by distinct electrocortical activities. Behav Brain Res 164:42-51.

Cardoso de Oliveira S, Thiele A, Hoffmann KP (1997) Synchronization of neuronal activity during stimulus expectation in a direction discrimination task. J Neurosci 17:9248-9260.

Casanova C, Freeman RD, Nordmann JP (1989) Monocular and binocular response properties of cells in the striate-recipient zone of the cat's lateral posterior-pulvinar complex. J Neurophysiol 62:544-557.

Casanova C, Savard T, Darveau S (1997) Contributon of area 17 to cell responses in the striate-recipient zone of the cat's lateral-posterior pulvinar complex. Eur J Neurosci 9:1026-1036.

Casanova C, Merabet L, Desautels A, Minville K (2001) Higher-order motion processing in the pulvinar. Prog Brain Res 134:71-82.

Chalupa LM (1991) Visual function of the pulvinar. In: Vision and visual dysfunction, Vol 4 (Leventhal AG, ed), pp 141-159. Boca Raton, FL: CRC.

Chalupa LM, Abramson BP (1988) Receptive-field properties in the tectoand striate-recipient zones of the cat's lateral-posterior nucleus. Prog Brain Res 75:85-94.

Chalupa LM, Abramson BP (1989) Visual receptive fields in the striaterecipient zone of the lateral posterior-pulvinar complex. J Neurosci 9:347-357.

Chalupa LM, Coyle RS, Lindsley DB (1976) Effect of pulvinar lesion on visual pattern discrimination in monkeys. J Neurophysiol 39:354-369.

Desimone R, Wessinger M, Thomas L, Schneider W (1990) Attentional control of visual perception: cortical and subcortical mechanisms. Cold Spring Harb Symp Quant Biol 55:963-971.

Dumbrava D, Faubert J, Casanova C (2001) Global motion integration in the cat's lateral posterior-pulvinar complex. Eur J Neurosci 13: $2218-2226$

Feig S, Harting JK (1998) Corticocortical communication via the thalamus: ultrastructural studies of corticothalamic projections from area 17 to the lateral posterior nucleus of the cat and inferior pulvinar nucleus of the owl monkey. J Comp Neurol 395:281-295.

Gail A, Brinksmeyer HJ, Eckhorn R (2004) Perception-related modulations of local field potential power and coherence in primary visual cortex of awake monkey during binocular rivalry. Cereb Cortex 14:300-313.

Garey LJ, Dreher B, Robinson SR (1991) The organization of visual thalamus. In: Vision and visual dysfunction, Vol 3 (Dreher B, Robinson SR, eds), pp 176-234. Houndmills, UK: Macmillan.

Gould JE, Chalupa LM, Lindsley DB (1974) Modification of pulvinar and geniculo-cortical evoked potentials during visual discrimination learning in monkeys. Electroencephalogr Clin Neurophysiol 36:639-649.

Grant S, Hilgetag CC (2005) Graded classes of cortical connections: quantitative analyses of laminar projections to motion areas of cat extrastriate cortex. Eur J Neurosci 22:681-696.

Grant S, Shipp S (1991) Visuotopic organization of the lateral suprasylvian area and of an adjacent area of the ectosylvian gyrus of cat cortex: a physiological and connectional study. Vis Neurosci 6:315-338.

Grieve K, Acuna C, Cudeiro J (2000) The primate pulvinar nuclei: vision and action. Trends Neurosci 23:35-39.

Gross J, Schmitz F, Schnitzler I, Kessler K, Shapiro K, Hommel B, Schnitzler A (2004) Modulation of long-range neural synchrony reflects temporal limitations of visual attention in humans. Proc Natl Acad Sci USA 101:13050-13055.

Guillery RW (1995) Anatomical evidence concerning the role of the thalamus in corticocortical communication: a brief review. J Anat 187:583-592.

Guillery RW, Sherman SM (2002) Thalamic relay functions and their role in corticocortical communication: generalizations from the visual system. Neuron 33:163-175. 
Guillery RW, Feig SL, Van Lieshout DP (2001) Connections of higher order visual relays in the thalamus: a study of corticothalamic pathways in cats. J Comp Neurol 438:66-85.

Harutiunian-Kozak BA, Hekmian AA, Dec K, Grigorian GE (1981) Responses of cat's pulvinar neurons to moving visual stimuli. Acta Neurobiol Exp 41:127-145.

Huppe-Gourgues F, Bickford ME, Boire D, Ptito M, Casanova C (2006) Distribution, morphology, and synaptic targets of corticothalamic terminals in the cat lateral posterior-pulvinar complex that originate from the posteromedial lateral suprasylvian cortex. J Comp Neurol 497:847-863.

Hutchins B, Updyke BV (1988) The lateral posterior complex of the cat: studies of the functional organization. Prog Brain Res 75:75-83.

Hutchins B, Updyke BV (1989) Retinotopic organization within the lateral posterior complex of the cat. J Comp Neurol 285:350-398.

Jones E (2001) The thalamic matrix and thalamocortical synchrony. Trends Neurosci 24:595-601.

Kelly LR, Li J, Carden WB, Bickford ME (2003) Ultrastructure and synaptic targets of tectothalamic terminals in the cat lateral posterior nucleus. J Comp Neurol 464:472-486.

Kiefer W, Kruger K, Strauss G, Berlucchi G (1989) Considerable deficits in the detection performance of the cat after lesion of the suprasylvian visual cortex. Exp Brain Res 75:208-212.

Kopell N, Ermentrout GB, Whittington MA, Traub RD (2000) Gamma rhythms and beta rhythms have different synchronization properties. Proc Natl Acad Sci USA 97:1867-1872.

Liang H, Bressler SL, Ding M, Truccolo WA, Nakamura R (2002) Synchronized activity in prefrontal cortex during anticipation of visuomotor processing. NeuroReport 13:2011-2015.

Lindström S, Wróbel A (1990) Frequency dependent corticofugal excitation of principal cells in the cat's dorsal lateral geniculate nucleus. Exp Brain Res 79:313-318.

Llinas RR, Grace A, Yarom Y (1991) In vitro neurons in mammalian cortical layer 4 exhibit intrinsic oscillatory activity in the $10-$ to $50-\mathrm{Hz}$ frequency range. Proc Natl Acad Sci USA 88:897-901.

Maunsell JH (2004) Neuronal representation of cognitive state: reward or attention. Trends Cogn Sci 8:261-265.

Merabet L, Minville K, Ptito M, Casanova C (2000) Responses of neurons in the cat posteromedial lateral suprasylvian cortex to moving texture patterns. Neuroscience 97:611-623.

Miceli D, Reperant J, Marchand L, Ward R, Vesselkin N (1991) Divergence and collateral axon branching in subsystems of visual cortical projections from the cat lateral posterior nucleus. J Hirnforsch 32:165-173.

Montaron MF, Bouyer JJ, Rougeul-Buser A (1979) Relationship between attention and mu rhythms in the cat and the monkey. Rev Electroencephalogr Neurophysiol Clin 9:333-339.

Niebur E, Hsiao SS, Johnson KO (2002) Synchrony: a neuronal mechanism for attentional selection? Curr Opin Neurobiol 12:190-194.

Olshausen BA, Anderson CH, Van Essen DC (1993) A neurobiological model of visual attention and invariant pattern recognition based on dynamic routing of information. J Neuroscience 13:4700-4719.

Ouellette BG, Minville K, Faubert J, Casanova C (2004) Simple and complex visual motion response properties in the anterior medial bank of the lateral suprasylvian cortex. Neuroscience 123:231-245.

Payne BR, Lomber SG (2003) Quantitative analyses of principal and secondary compound parieto-occipital feedback pathways in cat. Exp Brain Res 152:420-433.

Petersen SE, Robinson DL, Morris JD (1987) Contributions of the pulvinar to visual spatial attention. Neuropsychologia 25:97-105.

Portas CM, Rees G, Howseman AM, Josephs O, Turner R, Frith CD (1998) A specific role for the thalamus in mediating the interaction of attention and arousal in humans. J Neurosci 18:8979-8989.

Raczkowski D, Rosenquist AC (1983) Connections of the multiple visual cortical areas with the lateral posterior-pulvinar complex in the cat. J Neurosci 3:1912-1942.

Rauschecker JP, von Grunau MW, Poulin C (1987) Centrifugal organization of direction preference in the cat's lateral suprasylvian visual cortex and its relation to flow field processing. J Neurosci 7:943-958.

Robinson DL, Petersen SE (1992) The pulvinar and visual salience. Trends Neurosci 15:127-132.

Rudolph KK, Pasternak T (1996) Lesions in cat lateral suprasylvian cortex affect perception of complex motion. Cereb Cortex 6:814-822.

Sherman SM, Guillery RW (2002) The role of the thalamus in the flow of information to the cortex. Philos Trans R Soc Lond B Biol Sci 357:1695-1708.

Shipp S (2003) The functional logic of cortico-pulvinar connections. Philos Trans R Soc Lond B Biol Sci 358:1605-1624.

Shipp S (2004) The brain circuitry of attention. Trends Cogn Sci 8:223-230.

Shumikhina S, Molotchnikoff S (1995) Visually-triggered oscillation in the cat lateral posterior-pulvinar complex. NeuroReport 6:2341-2347.

Shumikhina S, Molotchnikoff S (1999) Pulvinar participates in synchronizing neural assemblies in the visual cortex, in cats. Neurosci Lett 272:135-139.

Shupert C, Cornwell P, Payne B (1993) Differential sparing of depth perception, orienting and optokinetic nystagmus after neonatal versus adult lesions of cortical areas 17, 18 and 19 in the cat. Behav Neurosci 107:633-650.

Stone J (1983) Parallel processing in the visual system: the classification of retinal ganglion cells and its impact on the neurobiology of vision. New York: Plenum.

Stone J, Dreher B (1973) Projection of X- and Y-cells of the cat's lateral geniculate nucleus to areas 17 and 18 of visual cortex. J Neurophysiol 36:551-567.

Symonds LL, Rosenquist AC, Edwards SB, Palmer LA (1981) Projections of the pulvinar-lateral posterior complex to visual cortical areas in the cat. Neuroscience 6:1995-2020.

Tallon-Baudry C, Bertrand O, Fischer C (2001) Oscillatory synchrony between human extrastriate areas during visual short-term memory maintenance. J Neurosci 21:RC177(1-5).

Tusa RJ, Palmer LA, Rosenquist AC (1981) Multiple cortical visual areas: visual field topography in the cat. In: Cortical sensory organization (Woolsey CN, ed), pp 1-31. Clifton, NJ: Humana.

Updyke BV (1977) Topographic organization of the projections from the cortical areas 17, 18 and 19 onto the thalamus, pretectum, and superior colliculus in the cat. J Comp Neurol 173:81-122.

Updyke BV (1983) A reevaluation of the functional organization and cytoarchitecture of the feline lateral posterior complex, with observations on adjoining cell groups. J Comp Neurol 219:143-181.

Varela F, Lachaux J-P, Rodriguez E, Martinerie J (2001) The brainweb: phase synchronization and large-scale integration. Nat Rev Neurosci 2:229-239.

von Stein A, Rappelsberger P, Sarnthein J, Petsche H (1999) Synchronization between temporal and parietal cortex during multimodal object processing in man. Cereb Cortex 9:137-150.

Waleszczyk W, Bekisz M, Wróbel A (2005) Cortical modulation of neuronal activity in the cat's lateral geniculate and perigeniculate nuclei. Exp Neurol 196:54-72.

Wróbel A (2000) Beta activity: a carrier for visual attention. Acta Neurobiol Exp (Wars) 60:247-260.

Wróbel A, Bekisz M, Kublik E, Waleszczyk W (1994) 20 Hz bursting beta activity in the cortico-thalamic system of visually attending cats. Acta Neurobiol Exp (Wars) 54:95-107.

Wróbel A, Ghazaryan A, Bekisz M, Smyda A (2005) Attention related beta activity in the lateral-posterior nucleus of the cat. Acta Neurobiol Exp (Wars) [Suppl] 65:101. 